\title{
Star coloring high girth planar graphs
}

\author{
Craig Timmons \\ Department of Mathematics \\ California State University San Marcos \\ San Marcos, CA 92096, USA \\ ctimmons@csusm.edu
}

Submitted: Nov 24, 2007; Accepted: Sep 22, 2008; Published: Sep 29, 2008

Mathematics Subject Classification: 05C15

\begin{abstract}
A star coloring of a graph is a proper coloring such that no path on four vertices is 2-colored. We prove that every planar graph with girth at least 9 can be star colored using 5 colors, and that every planar graph with girth at least 14 can be star colored using 4 colors; the figure 4 is best possible. We give an example of a girth 7 planar graph that requires 5 colors to star color.
\end{abstract}

Keywords: star coloring, planar graph coloring.

Mathematics Subject Classification: 05C15.

\section{Introduction}

Recall that a proper coloring of a graph is an assignment of colors to the vertices of the graph such that adjacent vertices are assigned different colors. A star coloring of a graph $G$ is a proper coloring such that no path on four vertices is 2-colored. A k-star coloring of a graph $G$ is a star coloring of $G$ using at most $k$ colors. The smallest $k$ such that $G$ has a $k$-star coloring is the star chromatic number of $G$.

In 1973 Grünbaum [5] introduced star colorings and acyclic colorings. An acyclic coloring is a proper coloring such that no cycle is 2-colored. Every star coloring is an acyclic coloring but star coloring a graph typically requires more colors than acyclically coloring the same graph. In general, many star coloring questions are not as well understood as their acyclic counterparts. For example, Borodin [3] proved that every planar graph can be acyclically 5-colored. This result is best possible and was conjectured by Grünbaum [5]. On the other hand, Albertson, Chappell, Kierstead, Kündgen, and Ramamurthi [1] proved that every planar graph can be star colored using 20 colors, and gave an example of a planar graph that requires 10 colors to star color; but this gap remains open. 
Planar graphs of high girth are typically easier to color in the sense that fewer colors are needed. For instance Grötzsch [6] proved that every planar graph of girth at least 4 can be properly colored using 3 colors. Borodin, Kostochka, and Woodall [4] proved that every planar graph of girth at least 5 can be acyclically colored using 4 colors, and every planar graph of girth at least 7 can be acyclically colored using 3 colors; the figure 3 is best possible.

Even under high girth assumptions, the upper bounds for star colorings are not as tight as the corresponding acyclic bounds. A result by Nešetřil and Ossona de Mendez [9] implies that every planar graph of girth at least 4 can be star colored using 18 colors; whereas Kierstead, Kündgen, and Timmons [7] gave an example of a bipartite planar graph that requires 8 colors to star color. Albertson et al. [1] proved that every planar graph of girth at least 5 can be star colored using 16 colors, every planar graph of girth at least 7 can be star colored with 9 colors, and planar graphs of sufficiently large girth can be star colored using 4 colors; but no specific bound on the girth requirement was given. They also gave an example of a planar graph of arbitrarily high girth that requires 4 colors to star color.

This paper improves upon the upper bounds for planar graphs of girth at least 9. In Section 2 we introduce relevant definitions and notation. In Section 3 we prove that every planar graph of girth at least 14 can be star colored using 4 colors. In Section 4 we prove that every planar graph of girth at least 9 can be star colored using 5 colors. In Section 5 we give an example of a planar graph of girth 7 that requires 5 colors to star color. In Section 6 we collect the current best known bounds and present some open problems.

\section{Preliminaries}

All graphs considered are loopless graphs without multiple edges. We denote the vertex set and edge set of a graph $G$ by $V(G)$ and $E(G)$ respectively. If $G$ is a planar graph with a fixed embedding, we denote the set of faces of $G$ by $F(G)$. The length of a face $f$, denoted $l(f)$, is the number of edges on the boundary walk of $f$. If $v$ is a vertex with degree $d$ then we say $v$ is a $d$-vertex. We will denote the degree of $v$ by $\operatorname{deg}(v)$. Degree 2 vertices will play a prominent role. If $v$ is a $d$-vertex adjacent to $k 2$-vertices, we say $v$ is a $d(k)$-vertex. A 1-vertex is also called a pendant vertex.

The neighborhood of a vertex $v$ is the set of all vertices in $V(G)$ that are adjacent to $v$. Vertices in the neighborhood of $v$ are the neighbors of $v$. The second neighborhood of a vertex $v$ is the set of all vertices in $V(G)-\{v\}$ that are adjacent to a neighbor of $v$. A vertex in the second neighborhood of $v$ is a second neighbor of $v$. A set $S \subset V(G)$ is independent if no two of its vertices are neighbors, and 2-independent if no two of its vertices are neighbors or second neighbors. If $S \subset V(G)$, then $G[S]$ is the subgraph of $G$ induced by $S$.

A path on $n$ vertices will be denoted by $P_{n}$. A cycle on $n$ vertices will be denoted by $C_{n}$. The graph obtained by adding a pendant vertex to each vertex of $C_{n}$ will be denoted by $C_{n}^{\prime}$. When $n$ is not divisible by 3 , it is easy to see that $C_{n}^{\prime}$ requires 4 colors to star color (see Example 5.3 in [1]). 
Proposition 2.1 There exist planar graphs of arbitrarily high girth that require 4 colors to star color.

\section{$3 \quad$ Girth 14 planar graphs}

Albertson et al. [1] use the idea of partitioning the vertices of a graph into a forest and a 2-independent set to obtain a star coloring. We use this idea to show that planar graphs of girth at least 14 can be star colored using 4 colors, matching the construction from Proposition 2.1.

Theorem 3.1 The vertices of a planar graph of girth at least 14 can be partitioned into two disjoint sets $I$ and $F$ such that $G[F]$ is a forest and $I$ is a 2-independent set in $G$.

It is easy to see that $G[F]$ can be 3 -star colored (in each component of $G[F]$, fix an arbitrary root and then give each vertex color 1,2 or 3 according as its distance from the root is 0,1 or 2 modulo 3 ). Now using a fourth color for $I$ gives a 4 -star coloring of $G$, so we immediately have:

Corollary 3.2 If $G$ is a planar graph of girth at least 14 then $G$ is 4 -star colorable.

\section{Proof of Theorem 3.1.}

Let $G$ be a minimal counterexample with the smallest number of vertices and give $G$ a fixed embedding in the plane. We may assume $G$ is connected and has minimum degree 2 since pendant vertices may be put in $F$.

Claim 1: $G$ has no 2(2)-vertex.

Suppose $x$ is a 2(2)-vertex in $G$ with neighbors $y$ and $z$. Consider a desired partition for $G-\{x, y, z\}$. We extend the partition to $G$ which provides the needed contradiction. If possible, put $x$ into $I$, and put $y$ and $z$ into $F$. If $x$ cannot be put into $I$, then a second neighbor of $x$ must be in $I$. Put $x, y$ and $z$ into $F$. $G[F]$ is acyclic as any new cycle must pass through both second neighbors of $x$, but one of these second neighbors is in $I$. This extends the desired partition to $G$, a contradiction.

Claim 2: $G$ has no 3(3)-vertex adjacent to two 2(1)-vertices.

Suppose $x$ is a 3(3)-vertex adjacent to 2(1)-vertices $y$ and $z$. Label the nearby vertices as indicated in Figure 3.1, where vertices depicted with $\circ$ may have other neighbors. Consider a desired partition for $G-\left\{x, x_{1}, y, y_{1}, z, z_{1}\right\}$. If possible, put $x$ into $I$, and put all other vertices into $F$. If $x$ cannot be put into $I$, then it must be that $x_{2} \in I$. If $y_{2} \in F$ then put $y$ into $I$, and put all other vertices into $F$. If $y_{2} \in I$ then put all vertices into $F$. This extends the desired partition to $G$, a contradiction. 


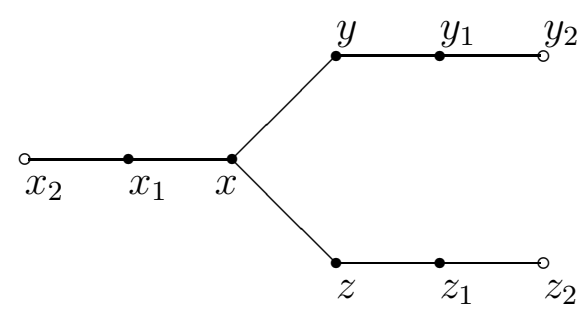

Figure 3.1: Claim 2

The proof is now finished by a simple discharging argument. Euler's Formula can be written in the form

$$
(12|E(G)|-14|V(G)|)+(2|E(G)|-14|F(G)|)=-28,
$$

which implies

$$
\sum_{v \in V(G)}(6 \operatorname{deg}(v)-14)+\sum_{f \in F(G)}(l(f)-14)=-28 .
$$

Since $G$ has girth $14, l(f) \geq 14$ for each $f \in F(G)$. This implies that the right sum is non-negative and so the left sum must be negative. For each vertex $v$ in $V(G)$, assign a charge of $6 \operatorname{deg}(v)-14$ to $v$. The charge is now redistributed according to the following rules:

1. Each 2(1)-vertex receives a charge of 2 from its neighbor of degree greater than 2.

2. Each 2(0)-vertex receives a charge of 1 from each neighbor.

The net charge of $V(G)$ after the redistribution is calculated. Let $v \in V(G)$.

Case 1: $v$ is a 2-vertex

By Claim 1, $v$ is not a 2(2)-vertex. If $v$ is a 2(1)-vertex, then by Rule $1, v$ receives charge 2. Since $v$ does not send out any charge, the charge of $v$ after redistribution is $6 \cdot 2-14+2=0$.

If $v$ is a $2(0)$-vertex, then by Rule $2, v$ receives charge 1 from each neighbor. Since $v$ does not send out any charge, the charge of $v$ after redistribution is $6 \cdot 2-14+1+1=0$.

Case 2: $v$ is a 3 -vertex

If $v$ is a 3(3)-vertex, then by Claim 2, $v$ is adjacent to at most one 2(1)-vertex. Then $v$ at most will send out charge 2 to one 2(1)-vertex, and charge 1 to each of its of other two neighbors. The charge of $v$ after redistribution is at least $6 \cdot 3-14-2-1-1=0$.

If $v$ is a $3(k)$-vertex with $k \leq 2$, then at most $v$ will send out charge $2 k$ to $k 2(1)$ vertices. The charge of $v$ after redistribution is at least $6 \cdot 3-14-2 k \geq 0$ as $k \leq 2$.

Case 3: $v$ has degree greater than 3

At most $v$ sends out charge $2 \operatorname{deg}(v)$. The charge of $v$ after redistribution is at least $6 \operatorname{deg}(v)-14-2 \operatorname{deg}(v)=4 \operatorname{deg}(v)-14 \geq 0 \operatorname{as} \operatorname{deg}(v) \geq 4$.

Cases 1-3 show that the charge of each vertex after redistribution is non-negative so that the net charge assigned to $V(G)$ is non-negative. This contradicts the fact that the net charge assigned to $V(G)$ is negative. Thus no such minimal counterexample exists. 


\section{Girth 9 planar graphs}

To prove that girth 9 planar graphs can be star colored with 5 colors, we use a similar approach as used for girth 14 planar graphs, except that the partition is into three sets.

Theorem 4.1 The vertices of a planar graph of girth at least 9 can be partitioned into three disjoint sets $F, I_{1}$ and $I_{2}$ such that $G[F]$ is a forest, $I_{1}$ is a 2-independent set in $G\left[F \cup I_{1}\right]$, and $I_{2}$ is a 2-independent set in $G$.

Corollary 4.2 If $G$ is a planar graph of girth at least 9 then $G$ is 5-star colorable.

Proof. Let $G$ be a planar graph with girth at least 9 , and consider the partition of $G$ given by Theorem 4.1. Star color the vertices in $F$ using colors 1, 2 and 3. Assign colors 4 and 5 to the vertices in $I_{1}$ and $I_{2}$ respectively. A potentially 2-colored $P_{4}$ cannot use color 5 since $I_{2}$ is a 2-independent set in $G$. Similarly it cannot use color 4 since $I_{1}$ is a 2-independent set in $G\left[F \cup I_{1}\right]$; and colors 1, 2 and 3 form a star coloring of $G[F]$.

\section{Proof of Theorem 4.1.}

Let $G$ be a minimal counterexample with the smallest number of vertices and give $G$ a fixed embedding in the plane. We may assume $G$ is connected and has minimum degree 2 .

Claim 1: $G$ has no 2(2)-vertex.

This follows as in Claim 1 of Theorem 3.1 by taking $I=I_{2}$.

Claim 2: $G$ has no 2(1)-vertex adjacent to a 3-vertex.

Suppose $x$ is a 2(1)-vertex adjacent to a 3-vertex $y$. Let $z$ be the 2 -vertex adjacent to $x$. Consider a desired partition for $G-\{x, z\}$. If $y \in I_{1} \cup I_{2}$, then put $x$ and $z$ into $F$; so assume $y \in F$. If possible, put $x$ into $I_{1} \cup I_{2}$ and put $z$ into $F$. Assume $x$ cannot be put into $I_{1} \cup I_{2}$. Then a second neighbor of $x$ must be in $I_{1}$, and another second neighbor of $x$ must be in $I_{2}$. Then $x$ and $z$ may be put into $F$ as any cycle created by adding vertices to $F$ must pass through two distinct second neighbors of $x$. This is impossible since $x$ only has three distinct second neighbors, two of which are in $I_{1} \cup I_{2}$. This extends the desired partition to $G$, a contradiction.

Claim 3: $G$ has no 3(3)-vertex.

Suppose $x$ is a 3(3)-vertex with neighbors $y, z$ and $t$. Consider a desired partition of the subgraph of $G$ obtained by removing $x$ and its neighbors. If possible, put $x$ into $I_{1} \cup I_{2}$ and put all other vertices into $F$. Assume $x$ cannot be put into $I_{1} \cup I_{2}$. Then a second neighbor of $x$ must be in $I_{1}$, and another second neighbor of $x$ must be in $I_{2}$. Then we may put all vertices into $F$ since any cycle created by adding vertices to $F$ must pass through two distinct second neighbors of $x$.

Claim 4: $G$ has no 3(2)-vertex adjacent to another 3(2)-vertex. 
Suppose $x$ and $y$ are adjacent 3(2)-vertices. Label the nearby vertices as indicated in Figure 4.1. Consider a desired partition for $G-\left\{x, x_{1}, x_{1}^{\prime}, y, y_{1}, y_{1}^{\prime}\right\}$.

Suppose $x_{2} \in I_{1} \cup I_{2}$. If possible, put $y$ into $I_{1} \cup I_{2}$ and put all other vertices into $F$. Assume $y$ cannot be put into $I_{1} \cup I_{2}$. Then $\left\{y_{2}, y_{2}^{\prime}\right\} \subset I_{1} \cup I_{2}$ and all vertices may be put into $F$.

Therefore $x_{2} \notin I_{1} \cup I_{2}$ so that $x_{2} \in F$. By symmetry, $x_{2}^{\prime}, y_{2}$ and $y_{2}^{\prime}$ must also be in $F$. Then we may put $x$ into $I_{1}, y$ into $I_{2}$, and all other vertices into $F$.

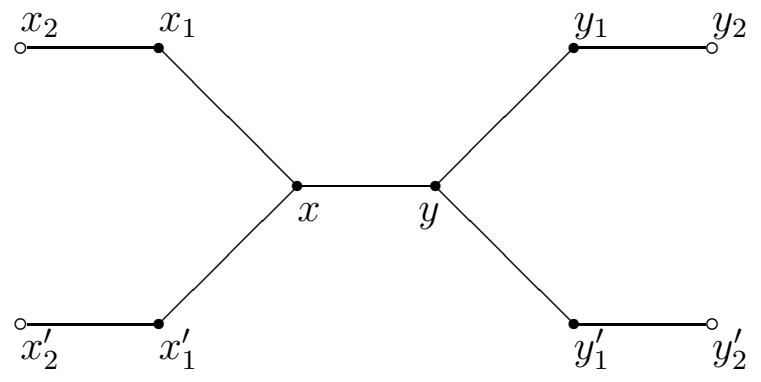

Figure 4.1: Claim 4

Claim 5: $G$ has no 3(1)-vertex adjacent to two 3(2)-vertices.

Suppose $x$ is a 3(1)-vertex adjacent to 3(2)-vertices $y$ and $z$. Label the nearby vertices as indicated in Figure 4.2. Consider a desired partition for $G-\left\{x, x_{1}, z, z_{1}, z_{1}^{\prime}, y, y_{1}, y_{1}^{\prime}\right\}$. If possible, put one of $y, z$ into $I_{1}$, put the other into $I_{2}$, and put all other vertices into $F$. Suppose this is not possible. Then we may assume $\left\{y_{2}, z_{2}\right\} \subset I_{1} \cup I_{2}$ or $z_{2} \in I_{1}, z_{2}^{\prime} \in I_{2}$.

First suppose $\left\{y_{2}, z_{2}\right\} \subset I_{1} \cup I_{2}$. Put $x$ into $I_{1}$ if $x_{2} \in I_{2}$, and into $I_{2}$ otherwise; and put all other vertices into $F$.

Now suppose $z_{2} \in I_{1}, z_{2}^{\prime} \in I_{2}$. If $y_{2}$ or $y_{2}^{\prime}$ is in $I_{1} \cup I_{2}$, then we are back in the previous case so assume $\left\{y_{2}, y_{2}^{\prime}\right\} \subset F$. Put $y$ into $I_{1}$, and put all other vertices into $F$.

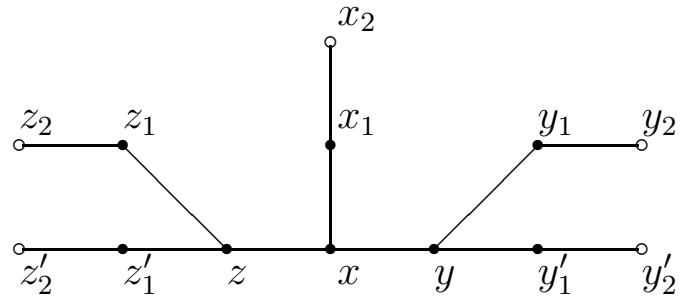

Figure 4.2: Claim 5

Claim 6: $G$ has no 4(4)-vertex adjacent to a 2(1)-vertex.

Suppose $x$ is a 4(4)-vertex adjacent to a 2(1)-vertex $y$. Consider a desired partition for the subgraph obtained by removing $x, y$, and their neighbors. If possible, put $x$ into $I_{1} \cup I_{2}$, and put all other vertices into $F$. Assume this is not possible. Then a second neighbor of $x$ must be in $I_{1}$ and another second neighbor of $x$ must be in $I_{2}$. We can put $y$ into one of $I_{1}, I_{2}$ since only one of $y$ 's second neighbors was not removed, and we put all other vertices into $F$. 
Definition 4.3 A weak $d(k)$-vertex is a $d(k)$-vertex all of whose degree 2 neighbors are $2(1)$-vertices.

Claim 7: $G$ has no weak 4(3)-vertex adjacent to a 3-vertex.

Suppose $x$ is a weak 4(3)-vertex adjacent to a 3 -vertex $y$. Label the nearby vertices as indicated Figure 4.3. Consider a desired partition for $G-\left\{x, x_{1}, x_{1}^{\prime}, x_{1}^{\prime \prime}, x_{2}, x_{2}^{\prime}, x_{2}^{\prime \prime}\right\}$. If possible put $x$ into $I_{1} \cup I_{2}$, and put all other vertices into $F$. Assume this is not possible. Then at least two of $y, y_{1}$ and $y_{1}^{\prime}$ must be in $I_{1} \cup I_{2}$; so assume $y_{1} \in I_{1} \cup I_{2}$.

If $y \in I_{1} \cup I_{2}$, then move $y$ into $F$. If $y_{1}^{\prime} \in F$, then $x$ may be put into one of $I_{1}, I_{2}$, and all other vertices may be put into $F$. Assume $y_{1}^{\prime} \in I_{1} \cup I_{2}$. Then any cycle obtained by adding vertices to $F$ must include at least one of $x_{1}, x_{1}^{\prime}$. If possible, put $x_{1}$ and $x_{1}^{\prime}$ into $I_{1} \cup I_{2}$, and put all other vertices into $F$. Otherwise $\left\{x_{3}, x_{3}^{\prime}\right\} \subset I_{1} \cup I_{2}$ and all vertices may be put into $F$.

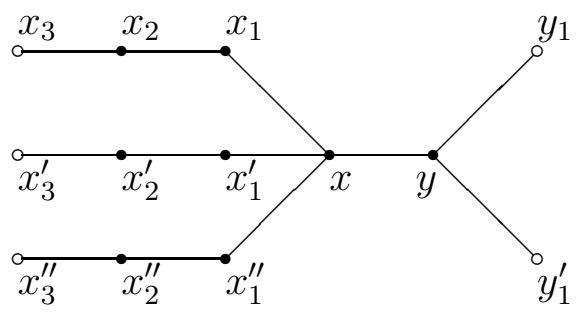

Figure 4.3: Claim 7

Claim 8: $G$ has no 4(3)-vertex adjacent to a 3(2)-vertex.

Suppose $x$ is a 4(3)-vertex adjacent to a 3(2)-vertex $y$. Label the nearby vertices as indicated in Figure 4.4. Consider a desired partition for $G-\left\{x, x_{1}, x_{1}^{\prime}, x_{1}^{\prime \prime}, y, y_{1}, y_{1}^{\prime}\right\}$. To show that the partition can be extended to $G$, we consider two cases.

Case 1: $x$ has at most one second neighbor in $I_{1} \cup I_{2}$.

Since $x$ has at most one second neighbor in $I_{1} \cup I_{2}, x$ can be put into one of $I_{1}, I_{2}$. If $y_{2}$ or $y_{2}^{\prime}$ is in $I_{1} \cup I_{2}$, then put all remaining vertices into $F$. Otherwise, $y_{2}$ and $y_{2}^{\prime}$ are both in $F$. Put $y$ into $I_{1}$ if $x \in I_{2}$, and $I_{2}$ otherwise; and put all remaining vertices into $F$.

Case 2: At least two second neighbors of $x$ are in $I_{1} \cup I_{2}$.

If $\left\{y_{2}, y_{2}^{\prime}\right\} \subset I_{1} \cup I_{2}$, then put all vertices into $F$. Otherwise, at least one of $y_{2}, y_{2}^{\prime}$ is in $F$ so that we may put $y$ into $I_{1} \cup I_{2}$, and all other vertices into $F$.

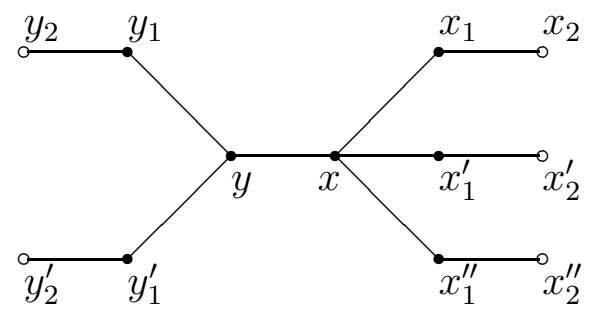

Figure 4.4: Claim 8 
Claim 9: $G$ has no 4(3)-vertex adjacent to a weak 4(3)-vertex.

Suppose $x$ is a 4(3)-vertex adjacent to a weak 4(3)-vertex $y$. Consider a desired partition for the subgraph of $G$ obtained by removing $y$, and its neighbors and second neighbors. If possible, put $x$ into $I_{1} \cup I_{2}$, put $y$ into $I_{1}$ if $x \in I_{2}$, and $I_{2}$ otherwise; and put all other vertices into $F$. Assume $x$ cannot be put into $I_{1} \cup I_{2}$. Then a second neighbor of $x$ is in $I_{1}$, and another second neighbor of $x$ is in $I_{2}$. Put $y$ into $I_{1}$ and put all other vertices into $F$.

Claim 10: $G$ has no weak 4(2)-vertex adjacent to two weak 4(3)-vertices.

Suppose $x$ is a weak 4(2)-vertex adjacent to two weak 4(3)-vertices $y$ and $z$. Consider a desired partition for the subgraph of $G$ obtained by removing $x, y$ and $z$, and all their neighbors and second neighbors. Put $x$ into $I_{2}$, put $y$ and $z$ into $I_{1}$, and put all other vertices into $F$. Note that $I_{1}$ is 2 -independent in $G\left[F \cup I_{1}\right]$, although it is not 2-independent in $G$.

Claim 11: $G$ has no weak 4(2)-vertex adjacent to a weak 4(3)-vertex and a 3(2)-vertex.

Suppose $x$ is a weak 4(2)-vertex adjacent to a weak 4(3)-vertex $y$ and a 3(2)-vertex $z$. Consider a desired partition for the subgraph of $G$ obtained by removing $x$ and $y$, and all their neighbors and second neighbors. If possible, put $z$ and $y$ into $I_{1}$, put $x$ into $I_{2}$, and put all other vertices into $F$. Assume it is not possible to put $z$ into $I_{1}$. Then a second neighbor of $z$ must be in $I_{1}$. Put $x$ into $I_{1}$, put $y$ into $I_{2}$, and put all other vertices into $F$.

Claim 12: $G$ has no 5(5)-vertex adjacent to four 2(1)-vertices.

Suppose $x$ is a 5(5)-vertex adjacent to four 2(1)-vertices and a 2-vertex $y$. Let $z$ be the neighbor of $y$ where $z \neq x$. Consider a desired partition for the subgraph of $G$ obtained by removing $x$, all of its neighbors and second neighbors except for $z$. Since only one second neighbor of $x$ was not removed, $x$ can be put into one of $I_{1}, I_{2}$, and we put all other vertices into $F$.

Claim 13: $G$ has no weak 5(4)-vertex adjacent to a weak 4(3)-vertex.

Suppose $x$ is a weak 5(4)-vertex adjacent to weak 4(3)-vertex $y$. Consider a partition for the subgraph of $G$ obtained by removing $x$ and $y$, and all their neighbors and second neighbors. Put $x$ into $I_{1}$, put $y$ into $I_{2}$, and put all other vertices into $F$.

The proof is now finished by a discharging argument. Euler's formula can be written in the form

$$
(14|E(G)|-18|V(G)|)+(4|E(G)|-18|F(G)|)=-36,
$$

which implies

$$
\sum_{v \in V(G)}(7 \operatorname{deg}(v)-18)+\sum_{f \in F(G)}(2 l(f)-18)=-36 .
$$

Since $G$ has girth $9, l(f) \geq 9$ for each face $f \in F(G)$. This implies that the right sum is non-negative and so the left sum must be negative. For each vertex $v$ in $V(G)$, assign 
a charge of $7 \operatorname{deg}(v)-18$ to $v$. The charge is now redistributed according to the following rules:

1. Each 2(0)-vertex receives a charge of 2 from each neighbor.

2. Each 2(1)-vertex receives a charge of 4 from the neighbor of degree greater than two.

3. Each 3(2)-vertex receives a charge of 1 from the neighbor of degree greater than two.

4. Each weak 4(3)-vertex receives a charge of 2 from the neighbor of degree greater than two.

The net charge of $V(G)$ after the redistribution is calculated. Let $v \in V(G)$.

Case 1: $v$ is a 2-vertex

By Claim 1, $v$ is not a 2(2)-vertex. If $v$ is a $2(1)$-vertex, then $v$ receives charge 4 from its neighbor of degree greater than two and $v$ does not send out any charge. The charge of $v$ after redistribution is $7 \cdot 2-18+4=0$. If $v$ is a 2(0)-vertex, then $v$ receives charge 2 from each neighbor and $v$ does not send out any charge. The charge of $v$ after redistribution is $7 \cdot 2-18+2+2=0$.

Case 2: $v$ is a 3 -vertex

By Claim 2, $v$ is not adjacent to a 2(1) vertex. By Claim 7, $v$ is not adjacent to a weak $4(3)$-vertex. Thus $v$ will only send charge to 2(0)-vertices and 3(2)-vertices. By Claim 3, $v$ is not a $3(3)$-vertex.

If $v$ is a 3(2)-vertex, then $v$ sends out charge 4 to two 2(0)-vertices and receives charge 1 from its neighbor of degree greater than two. By Claim $4, v$ will not send out any charge to another 3(2)-vertex. The charge of $v$ after redistribution is $7 \cdot 3-18-4+1=0$.

If $v$ is a 3(1)-vertex, then $v$ sends out charge 2 to a 2(0)-vertex and by Claim $5, v$ will send out at most charge 1 to a $3(2)$-vertex. The charge of $v$ after redistribution is at least $7 \cdot 3-18-2-1=0$.

If $v$ is a $3(0)$-vertex then at most $v$ will send out charge 3 to three $3(2)$-vertices. The charge of $v$ after redistribution is at least $7 \cdot 3-18-3=0$.

Case 3: $v$ is a 4 -vertex

If $v$ is a 4(4)-vertex then by Claim $6, v$ is not adjacent to a 2(1)-vertex. Therefore $v$ sends out charge 8 to four 2(0)-vertices. The charge of $v$ after redistribution is $7 \cdot 4-18-8=$ 2 .

If $v$ is a 4(3)-vertex then we consider three subcases.

Subcase 3.1a: $v$ is adjacent to three 2(1)-vertices i.e. $v$ is a weak 4(3)-vertex

By Rule 2, $v$ sends charge 12 to three 2(1)-vertices. By Claim 7, $v$ is not adjacent to a 3 -vertex so that $v$ does not send any charge to a 3(2)-vertex. By Claim 9, $v$ is not adjacent to a weak 4(3)-vertex so that $v$ does not send any charge to a weak 4(3)-vertex. By Rule 4, $v$ receives charge 2 from its neighbor of degree greater than two. The charge of $v$ after redistribution is $7 \cdot 4-18-12+2=0$.

Subcase 3.1b: $v$ is adjacent to two 2(1)-vertices and a 2(0)-vertex 
By Claim 8, $v$ is not adjacent to a 3(2)-vertex so that $v$ does not send any charge to a 3(2)-vertex. By Claim 9, $v$ is not adjacent to a weak 4(3)-vertex so that $v$ does not send any charge to a weak 4(3)-vertex. The charge of $v$ after redistribution is $7 \cdot 4-18-4-4-2=0$

Subcase 3.1c: $v$ is adjacent to at most one 2(1)-vertex

In this case, $v$ will at most send out charge 4 to a 2(1)-vertex and at most charge 2 to each of its remaining neighbors. The charge of $v$ after redistribution is at least $7 \cdot 4-18-4-2-2-2=0$.

If $v$ is a 4(2)-vertex then we consider two subcases.

Subcase 3.2a: $v$ is adjacent to two 2(1)-vertices

By Claim 10, $v$ is not adjacent to two weak 4(3)-vertices. By Claim 11, $v$ is not adjacent to a weak 4(3)-vertex and a 3(2)-vertex. Suppose $v$ is adjacent to a weak 4(3)vertex. Then $v$ sends charge 8 to two 2(1)-vertices, and charge 2 to a weak 4(3)-vertex. The charge of $v$ after redistribution is $7 \cdot 4-18-8-2=0$. Now suppose $v$ is not adjacent to a weak 4(3)-vertex. Then $v$ may be adjacent to two 3(2)-vertices. The charge of $v$ after redistribution is at least $7 \cdot 4-18-8-1-1=0$.

Subcase 3.2b: $v$ is adjacent to at most one 2(1)-vertex

In this case, $v$ will send out charge of at most 4 to a 2(1)-vertex, and at most 2 to each of its other three neighbors. The charge of $v$ after redistribution is at least $7 \cdot 4-18-4-6=0$.

If $v$ is a 4(1)-vertex, then $v$ sends out charge of at most 4 to a 2(1)-vertex and at most 2 to each of its other three neighbors. The charge of $v$ after redistribution is at least $7 \cdot 4-18-4-6=0$.

If $v$ is a 4(0)-vertex, then $v$ sends out charge of at most 8 to four weak 4(3)-vertices. The charge of $v$ after redistribution is at least $7 \cdot 4-18-8=2$.

Case 4: $v$ is a 5 -vertex

If $v$ is a 5(5)-vertex, then by Claim 12, $v$ is adjacent to at most three 2(1)-vertices. Therefore $v$ will send out charge of at most 12 to three 2(1)-vertices, and at most 4 to two other vertices. The charge of $v$ after redistribution is at least $7 \cdot 5-18-12-4=1$.

If $v$ is a $5(4)$-vertex then we consider two subcases.

Subcase 4.1: $v$ is a weak 5(4)-vertex.

By Claim 13, $v$ is not adjacent to a weak 4(3)-vertex so that $v$ will send out charge of at most 16 to four 2(1)-vertices, and at most 1 to a 3(2)-vertex. The charge of $v$ after redistribution is at least $7 \cdot 5-18-16-1=0$.

Subcase 4.2: $v$ is not a weak 5(4)-vertex

By definition, $v$ is adjacent to at most three 2(1)-vertices, and $v$ will send out charge at most 2 to each remaining neighbor. The charge of $v$ after redistribution is at least $7 \cdot 5-18-12-2-2=1$.

If $v$ is a $5(k)$-vertex with $k \leq 3$, then $v$ sends out charge at most $4 k$ to $k 2(1)$-vertices, and at most $(5-k) \cdot 2$ to its other neighbors. The charge of $v$ after redistribution is at least $7 \cdot 5-18-4 k-(5-k) \cdot 2 \geq 7 \cdot 5-18-12-4=1$ as $k \leq 3$.

Case 5: $v$ is a vertex of degree greater than 5

At most $v$ will send out charge $4 \operatorname{deg}(v)$. The charge of $v$ after redistribution is at least $7 \operatorname{deg}(v)-18-4 \operatorname{deg}(v)=3 \operatorname{deg}(v)-18 \geq 0$ as $\operatorname{deg}(v) \geq 6$. 
Cases 1-5 show that the charge of each vertex after redistribution is non-negative so that the net charge assigned to $V(G)$ is non-negative. This contradicts the fact that the net charge assigned to $V(G)$ is negative. Thus no such minimal counterexample exists.

\section{A construction}

In this section we give an example of a planar graph of girth 7 that requires 5 colors to star color. We begin with two definitions that play a key role in the construction.

Definition 5.1 A $k$-cluster with center $v$ is a graph $C$ together with a star coloring $f$ such that:

1. $C$ has vertex set $\left\{v, x_{1}, x_{2}, \ldots, x_{k}, x_{1}^{\prime}, x_{2}^{\prime}, \ldots, x_{k}^{\prime}\right\}$ where the $x_{i}^{\prime}$ 's need not be distinct;

2. $v$ has $k$ distinct neighbors $x_{1}, x_{2}, \ldots, x_{k}$;

3. each neighbor $x_{i}$ of $v$ is adjacent to a vertex $x_{i}^{\prime} \neq v$ with $f\left(x_{i}^{\prime}\right)=f(v)$.

Call the $k$ neighbors of $v$ the special neighbors of $v$. The edge $x_{i} x_{i}^{\prime}$ is said to be a leg of the $k$-cluster.

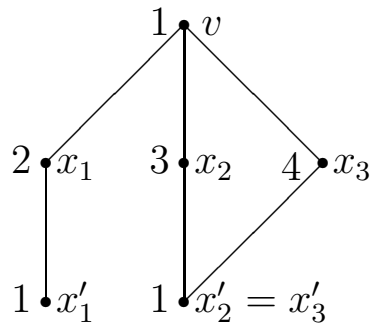

Figure 5.1: A 3-cluster with center $v$ and $\operatorname{legs} x_{1} x_{1}^{\prime}, x_{2} x_{2}^{\prime}$ and $x_{3} x_{3}^{\prime}$

$k$-clusters are useful for our purposes as they can forbid colors to appear on neighbors of the center of the cluster that are not contained in the cluster. For example, if $v$ is as in Figure 5.1, then any neighbor of $v$ not in 3-cluster cannot be assigned colors 1, 2, 3 or 4 without creating a 2 -colored $P_{4}$ or an improper coloring. Thus we make use of $k$-clusters by attaching them to existing graphs in a specific fashion. The center of the $k$-cluster is identified with a vertex in the graph, but the other vertices in the $k$-cluster are new vertices not contained in the vertex set of the graph. We formalize this idea in the next definition.

Definition 5.2 Let $v$ be the center of a $k$-cluster $C$ with $\operatorname{legs} x_{1} x_{1}^{\prime}, \ldots, x_{k} x_{k}^{\prime}$. Let $G$ be a graph and let $v$ be a vertex in $G$ with $x_{1}, x_{2}, \ldots, x_{k}, x_{1}^{\prime}, x_{2}^{\prime}, \ldots, x_{k}^{\prime}$ not in $G$. Attaching $C$ to $v$ in $G$ results in a graph with vertex set $V(G) \cup V(C)$, where $V(G) \cap V(C)=\{v\}$, and edge set $E(G) \cup E(C)$, in which $V(C)$ is colored as in $C$. 
A 1-cluster attached to $x$ and a 1-cluster attached to $y$ in the graph $H_{1}$ can be seen in Figure 5.2.

We now proceed to the construction. First we construct a graph $G_{2}$ such that any 4-star coloring of $G_{2}$ contains a 2-cluster. We then construct a graph $G_{3}$ such that if a 2-cluster is attached to $G_{3}$, then a coloring of the 2-cluster cannot be extended to a 4-star coloring of $G_{3}$. Lastly we construct $G$ using $G_{2}$ and $\left|V\left(G_{2}\right)\right|$ copies of $G_{3}$, where each copy of $G_{3}$ is associated with a vertex of $G_{2}$. Then in any 4-star coloring of $G$, the induced copy of $G_{2}$ contains a 2-cluster, say with center $v$, and the copy of $G_{3}$ associated with $v$ cannot be colored.

$G_{2}$ will be constructed from copies of two smaller graphs $H_{1}$ and $H_{2}$. We first prove three lemmas regarding these smaller graphs before showing that any 4-star coloring of $G_{2}$ contains a 2 -cluster.

Let $b_{1}, b_{2}, \ldots, b_{10}$ be the vertices of $P_{10}$, and let $x$ and $y$ be two isolated vertices. For $i \in\{1,4,7,10\}$ add edges $x b_{i}$ and $y b_{i}$, and subdivide $x b_{i}$ and $y b_{i}$ with $a_{i}$ and $c_{i}$ respectively. Call this graph $H_{1}$. Later in the construction of $H_{2}$, we will add copies of $H_{1}$ between two specific vertices. When we add a copy of $H_{1}$ between two vertices, say $u$ and $w$, we are identifying $u$ and $w$ with $x$ and $y$ respectively.

Lemma 5.3 Let $f$ be a 4-star coloring of $H_{1}$ such that $f\left(a_{i}\right)=f\left(a_{i+3}\right)$ and $f\left(c_{i}\right)=$ $f\left(c_{i+3}\right)$ for some $i \in\{1,4,7\}$. Then $H_{1}$ contains a 2-cluster.

Proof. If $f\left(a_{i}\right)=f\left(c_{i}\right)$, then $a_{i}$ is the center of a 2-cluster with legs $x a_{i+3}$ and $b_{i} c_{i}$; so assume $f\left(a_{i}\right) \neq f\left(c_{i}\right)$. If any of $b_{i}, b_{i+1}, b_{i+2}, b_{i+3}$ receives color $f\left(a_{i}\right)$ or $f\left(c_{i}\right)$, then we have either an improper coloring or a 2-cluster centered at one of $a_{i}, c_{i}, a_{i+3}, c_{i+3}$. However, it is not possible to star color the path induced by $b_{i}, b_{i+1}, b_{i+2}$ and $b_{i+3}$ with only two colors.

Lemma 5.4 Attach a 1-cluster to $x$ and a 1-cluster to $y$ in $H_{1}$ with legs $x_{1} x_{1}^{\prime}$ and $y_{1} y_{1}^{\prime}$ respectively. Let $f$ be a 4-star coloring of the clusters such that $f(x)=f(y)$. Then $f$ cannot be extended to a 4-star coloring of $H_{1}$ without creating a 2-cluster.

Proof. Suppose $f$ can be extended to a 4-star coloring of $H_{1}$ without creating a 2-cluster. Let $f(x)=f(y)=1$ and $f\left(x_{1}\right)=2$ where $x_{1}$ is the special neighbor of $x$ (see Figure 5.2). Observe that for each $i \in\{1,4,7,10\}, f\left(a_{i}\right) \notin\{1,2\}$ and $f\left(c_{i}\right) \notin\left\{1, f\left(y_{1}\right)\right\}$ where $y_{1}$ is the special neighbor of $y$.

Suppose $f\left(a_{i}\right)=f\left(a_{i+3}\right)=3$ with $i \in\{1,4,7\}$. If $f\left(c_{i}\right)=3$, then $a_{i}$ is the center of a 2-cluster with legs $b_{i} c_{i}$ and $x a_{i+3}$. Similarly, $f\left(c_{i+3}\right) \neq 3$. By Lemma 5.3, $f\left(c_{i}\right) \neq f\left(c_{i+3}\right)$ so assume $f\left(c_{i}\right)=2$ and $f\left(c_{i+3}\right)=4$. If $f\left(b_{i}\right)=1$ or $f\left(b_{i+3}\right)=1$, then $x$ is the center of a 2-cluster. Therefore $f\left(b_{i}\right)=4$ and $f\left(b_{i+3}\right)=2$. If $f\left(b_{i+1}\right)=2$, then $b_{i+1}$ is the center of a 2-cluster with legs $b_{i} c_{i}$ and $b_{i+2} b_{i+3}$. If $f\left(b_{i+1}\right)=3$, then $a_{i}$ is the center of a 2-cluster with legs $b_{i} b_{i+1}$ and $x a_{i+3}$. Thus $f\left(b_{i+1}\right)=1$ and similarly, $f\left(b_{i+2}\right)=1$ and we have an improper coloring.

Hence $f\left(a_{i}\right) \neq f\left(a_{i+3}\right)$ and so we may assume $f\left(a_{1}\right)=f\left(a_{7}\right)=3$ and $f\left(a_{4}\right)=f\left(a_{10}\right)=$ 4. Observe that if $f\left(a_{i}\right)=f\left(c_{i}\right)$ for some $i \in\{1,4,7,10\}$ then $a_{i}$ is the center of a 2-cluster. 
Suppose $f\left(y_{1}\right)=2$. By a similar argument as used in the previous paragraph, we must have $f\left(c_{1}\right)=f\left(c_{7}\right)=4$ and $f\left(c_{4}\right)=f\left(c_{10}\right)=3$. This forces $f\left(b_{4}\right)=f\left(b_{7}\right)=2$. If $f\left(b_{5}\right)=3$ or $f\left(b_{5}\right)=4$, then $c_{4}$ or $a_{4}$, respectively, is the center of a 2-cluster; so $f\left(b_{5}\right)=1$. Similarly, $f\left(b_{6}\right)=1$ and we have an improper coloring. Therefore $f\left(y_{1}\right) \neq 2$ and so we may assume $f\left(y_{1}\right)=3$. Then we must have $f\left(c_{1}\right)=f\left(c_{7}\right)=4$ and $f\left(c_{4}\right)=f\left(c_{10}\right)=2$, so that $f\left(b_{1}\right)=2$ and $f\left(b_{4}\right)=3$. If $f\left(b_{2}\right)=3$ then $b_{2}$ is the center of a 2-cluster, and if $f\left(b_{2}\right)=4$ then $c_{1}$ is the center of a 2 -cluster. Thus $f\left(b_{2}\right)=1$, and similarly $f\left(b_{3}\right)=1$ and we have an improper coloring.

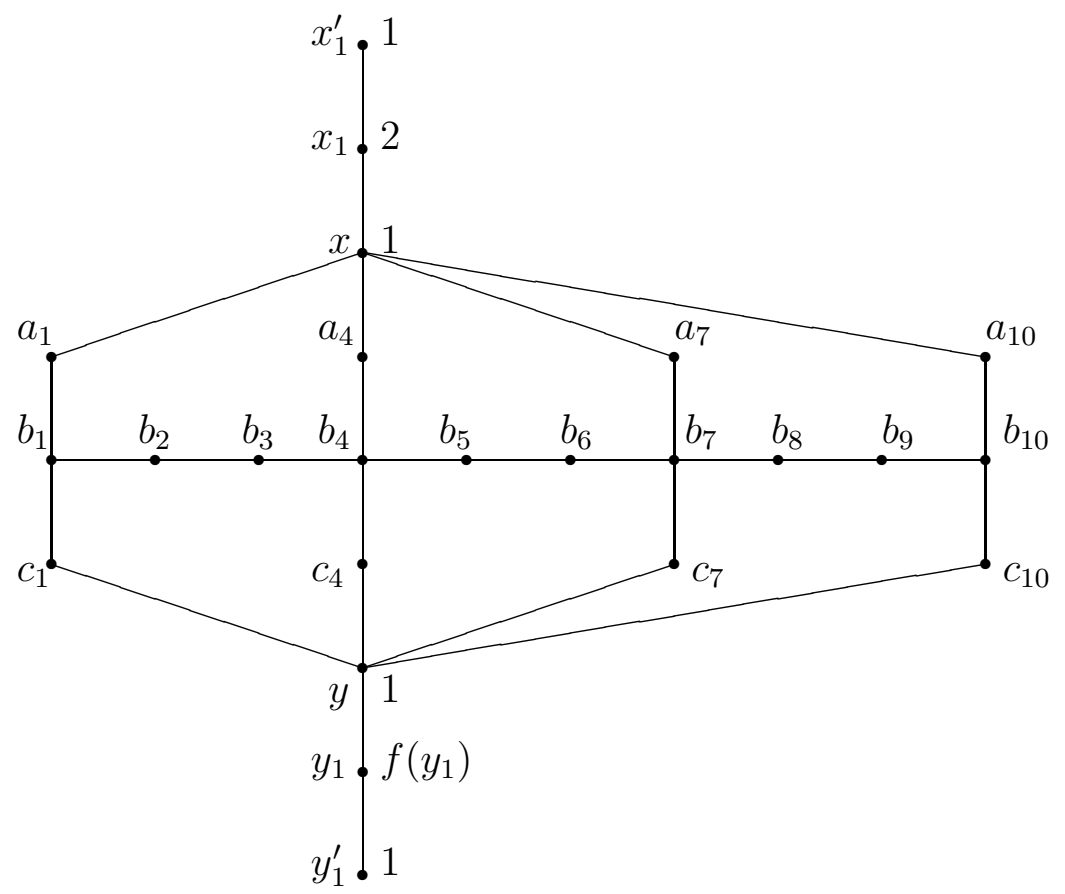

Figure 5.2: Lemma 5.4, 1-clusters attached to $x$ and $y$ in $H_{1}$

We now construct $H_{2}$. Let $b_{1}, b_{2}, \ldots, b_{22}$ be the vertices of $P_{22}$, and let $x$ and $y$ be two isolated vertices. For $i \equiv 1$ modulo 3 , add edges $x b_{i}$ and $y b_{i}$, and subdivide $x b_{i}$ and $y b_{i}$ with $a_{i}$ and $c_{i}$ respectively. For $i \equiv 0,2$ modulo 3 , add copies of $H_{1}$ between $x$ and $b_{i}$, and between $y$ and $b_{i}$. Call this graph $H_{2}$. In the construction of $G_{2}$ we will add copies of $H_{2}$ between two specific vertices. When we add a copy of $H_{2}$ between two vertices, say $u$ and $w$, we are identifying $u$ and $w$ with $x$ and $y$ respectively.

Lemma 5.5 Attach a 1-cluster to $x$ and a 1-cluster to $y$ in $H_{2}$ with legs $x_{1} x_{1}^{\prime}$ and $y_{1} y_{1}^{\prime}$ respectively. Let $f$ be a 4-star coloring of the clusters such that $f\left(x_{1}\right)=f\left(y_{1}\right)$. Then $f$ cannot be extended to a 4-star coloring of $\mathrm{H}_{2}$ without creating a 2-cluster.

Proof. Suppose $f$ can be extended to a 4-star coloring of $H_{2}$ without creating a 2-cluster. If $f(x)=f(y)$ then since $x$ and $y$ have an induced copy of $H_{1}$ between them, we may apply Lemma 5.4 to this copy of $H_{1}$. Assume $f(x) \neq f(y)$, say $f(x)=1, f(y)=3$, and $f\left(x_{1}\right)=f\left(y_{1}\right)=2$. 
Suppose $f\left(a_{i}\right)=f\left(a_{j}\right)=4$ for some $i<j$ with $\{i, j\} \subset\{4,7,10,13,16,19\}$. If $f\left(b_{i}\right)=1$ or $f\left(b_{i}\right)=3$ then $x$ or $y$, respectively, is the center of a 2-cluster; so $f\left(b_{i}\right)=2$. Similarly, $f\left(b_{j}\right)=2$. If $f\left(c_{i}\right)=4$ then $a_{i}$ is the center of a 2-cluster with legs $b_{i} c_{i}$ and $x a_{j}$; so $f\left(c_{i}\right)=1$, and similarly $f\left(c_{j}\right)=1$. If $f\left(b_{i-1}\right)=1$ or $f\left(b_{i+1}\right)=1$, then $c_{i}$ is the center of a 2-cluster, and if $f\left(b_{i-1}\right)=4$ or $f\left(b_{i+1}\right)=4$ then $a_{i}$ is the center of a 2-cluster; so $f\left(b_{i-1}\right)=3$ and $f\left(b_{i+1}\right)=3$. Since $b_{i+1}$ and $y$ are centers of 1-clusters with legs $b_{i} b_{i-1}$ and $y_{1} y_{1}^{\prime}$, respectively, and $f\left(b_{i+1}\right)=f(y)$, we can apply Lemma 5.4 to the induced copy of $H_{1}$ between $b_{i+1}$ and $y$.

Hence we may assume that all but at most one $a_{i}$ with $i \in\{4,7,10,13,16,19\}$ receives color 3. Similarly, all but at most one $c_{i}$ with $i \in\{4,7,10,13,16,19\}$ receives color 1 . But there are six vertices $a_{i}$ and six vertices $c_{i}$ with $i \in\{4,7,10,13,16,19\}$. So there is a $j$ such that $f\left(a_{j}\right)=f\left(a_{j+3}\right)=3$ and $f\left(c_{j}\right)=f\left(c_{j+3}\right)=1$ and we may apply Lemma 5.3 to the copy of $H_{1}$ induced by $x, y, a_{j+k}, c_{j+k}, b_{j+l}$ where $k \in\{-3,0,3,6\}$ and $l \in\{-3,-2, \ldots, 5,6\}$.

We now construct $G_{2}$. Let $u_{1}, \ldots, u_{7}$ be the vertices on the 7-cycle in $C_{7}^{\prime}$ (the graph obtained by adding a pendant vertex to each vertex on $C_{7}$ ). For $1 \leq i \leq 7$, let $u_{i+7}$ be the pendant vertex adjacent to $u_{i}$. Add an isolated vertex $x$. For $1 \leq i \leq 14$, add five pendant vertices $u_{i}^{1}, \ldots, u_{i}^{5}$ to $u_{i}$, and add five pendant vertices $x_{i}^{1}, \ldots, x_{i}^{5}$ to $x$ (At this point $\operatorname{deg}\left(u_{i}\right)=8$ for $1 \leq i \leq 7, \operatorname{deg}\left(u_{i}\right)=6$ for $8 \leq i \leq 14$, and $\left.\operatorname{deg}(x)=70\right)$. For each $i, j$, add a copy of $H_{2}$ between $u_{i}^{j}$ and $x_{i}^{j}$. This completes the construction of $G_{2}$. It is easy to see that $G_{2}$ is planar.

Lemma 5.6 Any 4-star coloring of $G_{2}$ must contain a 2-cluster.

Proof. Let $f$ be a 4 -star coloring of $G_{2}$ and assume $f(x)=1$. Since $C_{7}^{\prime}$ requires 4 colors to star color, there is an $i \in\{1,2, \ldots, 14\}$ such that $f\left(u_{i}\right)=1$. Observe $\left\{f\left(u_{i}^{j}\right), f\left(x_{i}^{j}\right)\right\} \subset$ $\{2,3,4\}$ for $1 \leq j \leq 5$.

If there exists a $k \neq j$ such that $f\left(u_{i}^{j}\right)=f\left(u_{i}^{k}\right)$, then $u_{i}^{j}$ is the center of a 1-cluster with leg $u_{i} u_{i}^{k}$; and there are at most two values of $j$ for which there does not exist such a $k$. Similarly, there are at most two values of $j$ for which $x_{i}^{j}$ is not the center of a 1-cluster with special neighbor $x$. Thus there is at least one $j$ for which both $u_{i}^{j}$ and $x_{i}^{j}$ are centers of 1-clusters with special neighbors $u_{i}$ and $x$ respectively. By Lemma 5.5, the copy of $H_{2}$ between the centers of 1-clusters $u_{i}^{j}$ and $x_{i}^{j}$ must contain a 2-cluster.

$G_{3}$ is constructed in a similar fashion as $G_{2}$. Before constructing $G_{3}$, we need to construct a smaller graph $\mathrm{H}_{3}$, and prove a technical lemma.

Let $b_{1}, b_{2}, \ldots, b_{10}$ be the vertices of $P_{10}$, and let $x$ and $y$ be two isolated vertices. For each $i \in\{1, \ldots, 10\}$, add edges $x b_{i}$ and $y b_{i}$. For each $i \in\{1, \ldots, 10\}$, subdivide $x b_{i}$ twice with $a_{i}$ and $s_{i}$ so that $s_{i}$ is adjacent to $x$, and subdivide $y b_{i}$ twice with $c_{i}$ and $t_{i}$ so that $t_{i}$ is adjacent to $y$. Call this graph $H_{3}$. Later in the construction of $G_{3}$, we will add copies of $H_{3}$ between two specific vertices. When we add a copy of $H_{3}$ between two vertices, say $u$ and $w$, we are identifying $u$ and $w$ with $x$ and $y$ respectively. 
Lemma 5.7 Attach a 2-cluster to $x$ in $H_{3}$ with legs $x_{1} x_{1}^{\prime}, x_{2} x_{2}^{\prime}$ and attach a 2-cluster to $y$ in $H_{3}$ with legs $y_{1} y_{1}^{\prime}, y_{2} y_{2}^{\prime}$ where $y_{1}=x$. Let $f$ be a 4-star coloring of the clusters such that $f(y) \notin\left\{f(x), f\left(x_{1}\right), f\left(x_{2}\right)\right\}$. Then $f$ cannot be extended to a 4-star coloring of $\mathrm{H}_{3}$.

Proof. Suppose $f$ can be extended to a 4-star coloring of $H_{3}$. Let $f(x)=1, f\left(x_{1}\right)=$ $f\left(y_{2}\right)=2, f\left(x_{2}\right)=3$, and $f(y)=4$. Since $x$ and $y$ are both 2-clusters, we must have $f\left(s_{i}\right)=4$ and $f\left(t_{i}\right)=3$ for all $i$. An important observation is that only colors 2 and 3 are available for each $a_{i}$, and only colors 1 and 2 are available for each $c_{i}$.

First we show $f\left(b_{i}\right) \neq 2$ for $i \in\{4,5,6,7\}$. Suppose $f\left(b_{i}\right)=2$ and $f\left(b_{i+1}\right)=1$ for some $i \in\{4,5,6,7\}$. Then $f\left(c_{i}\right)=1$ and $f\left(c_{i+1}\right)=2$; but then $c_{i} b_{i} b_{i+1} c_{i+1}$ is 2-colored. Similarly, if $f\left(b_{i}\right)=2$ and $f\left(b_{i+1}\right)=3$, then $f\left(a_{i}\right)=3, f\left(a_{i+1}\right)=2$ and $a_{i} b_{i} b_{i+1} a_{i+1}$ is 2-colored. Therefore, if $f\left(b_{i}\right)=2$, we must have $f\left(b_{i-1}\right)=f\left(b_{i+1}\right)=4$. This forces $f\left(a_{i+1}\right)=3$ and $f\left(c_{i+1}\right)=1, f\left(b_{i+2}\right)=1$, and $f\left(c_{i+2}\right)=2$. Clearly $f\left(b_{i+3}\right) \neq 1$, and if $f\left(b_{i+3}\right)=4$ then $c_{i+1} b_{i+1} b_{i+2} b_{i+3}$ is 2-colored. If $f\left(b_{i+3}\right)=2$ then $f\left(c_{i+3}\right)=1$ and $c_{i+2} b_{i+2} b_{i+3} c_{i+3}$ is 2-colored; so $f\left(b_{i+3}\right)=3$. This forces $f\left(a_{i+3}\right)=2$ but then $c_{i+3}$ cannot be colored. We conclude that $f\left(b_{i}\right) \neq 2$ whenever $i \in\{4,5,6,7\}$.

The path $b_{4} b_{5} b_{6} b_{7}$ requires 3 colors to star color so that $f\left(b_{i}\right)=3$ for some $i \in$ $\{4,5,6,7\}$. This forces $f\left(a_{i}\right)=2$ and $f\left(c_{i}\right)=1$. If $f\left(b_{i+1}\right)=2$ then $f\left(a_{i+1}\right)=3$ and $a_{i} b_{i} b_{i+1} a_{i+1}$ is 2 -colored; so $f\left(b_{i+1}\right)=4$. Similarly, $f\left(b_{i-1}\right)=4$. This forces $f\left(a_{i+1}\right)=2$, $f\left(c_{i+1}\right)=1, f\left(b_{i+2}\right)=1$, and $f\left(c_{i+2}\right)=2$. If $f\left(b_{i+3}\right)=2$ then $f\left(c_{i+3}\right)=1$; but then $c_{i+2} b_{i+2} b_{i+3} c_{i+3}$ is 2-colored. So $f\left(b_{i+3}\right)=3$ which forces $f\left(c_{i+3}\right)=2$; but then $a_{i+3}$ cannot be colored.

We now construct $G_{3}$. Let $u_{1}, \ldots, u_{7}$ be the vertices on the 7 -cycle in $C_{7}^{\prime}$ and let $u_{i+7}$ be the pendant vertex adjacent to $u_{i}$ for $1 \leq i \leq 7$. Add an isolated vertex $x$. For $i \in\{8, \ldots, 14\}$, add edges $x u_{i}$ and subdivide $x u_{i}$ with $w_{i}$. For $i \in\{8, \ldots, 14\}$, add a copy of $H_{3}$ between $x$ and $w_{i}$. This completes the construction of $G_{3}$.

Lemma 5.8 Attach a 2-cluster to $x$ in $G_{3}$ with special neighbors $x_{1}$ and $x_{2}$ and let $f$ be a 4-star coloring of the 2-cluster. Then $f$ cannot be extended to a 4-star coloring of $G_{3}$.

Proof. Suppose $f$ can be extended to a 4 -star coloring of $G_{3}$. Assume $f(x)=1, f\left(x_{1}\right)=2$ and $f\left(x_{2}\right)=3$. Observe that $f\left(w_{i}\right)=4$ for $i \in\{8, \ldots, 14\}$. Since $C_{7}^{\prime}$ requires 4 colors to star color, there is an $i \in\{1, \ldots 7\}$ with $f\left(u_{i}\right)=4$. Then $w_{i+7}$ is the center of a 2-cluster with legs $u_{i+7} u_{i}$ and $x w_{i+8}(i+8$ reduced modulo 7 if necessary). Observe $f\left(w_{i+7}\right) \notin\{1,2,3\}$ and that $x$ is a special neighbor of $w_{i+7}$. Therefore by Lemma 5.7, the induced copy of $H_{3}$ between $x$ and $w_{i+7}$ cannot be 4-star colored.

We now construct $G$ using $G_{2}$ and $\left|V\left(G_{2}\right)\right|$ copies of $G_{3}$. For each vertex $v$ in $G_{2}$, attach a copy of $G_{3}$ by identifying $x$ with $v$, where $x$ is as in the description of the construction of $G_{3}$.

Theorem $5.9 G$ is a planar graph of girth 7 that is not 4-star colorable. 
Proof. Suppose $f$ is a 4-star coloring of $G$. By Lemma 5.6, the induced copy of $G_{2}$ in $G$ must contain a 2-cluster, say with center $v$. By Lemma 5.8, the copy of $G_{3}$ attached to $v$ cannot be 4-star colored, a contradiction.

The above construction and argument depends heavily on the idea of a $k$-cluster. $k$ clusters are used in [7] to construct a bipartite planar graph that requires 8 colors to star color, and in [10] to construct a planar graph of girth 5 requiring 6 colors to star color.

\section{Known bounds and open problems}

The table below shows the current best known bounds for the star chromatic number for planar graphs of a given girth. The best known bound is given along with the corresponding reference. Bounds without reference are established in this paper.

\begin{tabular}{|c||c||c||}
\hline \multicolumn{1}{|c||}{ Girth } & \multicolumn{2}{c||}{ Best known bounds } \\
\hline$g$ & lower bound & upper bound \\
\hline 3 & $10[1]$ & $20[1]$ \\
4 & $8[7]$ & $18[9]$ \\
5 & $6[10]$ & $16[1]$ \\
6 & 5 & $8[8]$ \\
7 & 5 & $7[10]$ \\
8 & $4[1]$ & $6[10]$ \\
$9-13$ & $4[1]$ & 5 \\
$14+$ & $4[1]$ & 4 \\
\hline
\end{tabular}

Table 6.1: Best known bounds

Problem 1: Determine the smallest girth $g$ such that any planar graph of girth at least $g$ can be partitioned into a forest and a 2-independent set.

Theorem 3.1 shows that all planar graphs of girth at least 14 admit such a partition, while Theorem 5.9 gives an example of girth 7 graph that does not admit such a partition. We believe that girth 14 is too high and that Theorem 3.1 can be improved.

Problem 2: Determine the smallest girth $g$ such that any planar graph of girth at least $g$ can be star colored with 4 colors.

Corollary 3.2 shows that planar graphs of girth at least 14 can be star colored with 4 colors, while Theorem 5.9 shows that there is a girth 7 planar graph that requires 5 colors to star color. We believe that any planar graph with girth at least 8 can be star colored with 4 colors.

Problem 3: Determine the smallest $k$ such that any planar graph has a star coloring with $k$ colors.

While Corollary 4.2, Corollary 3.2, and [10] improve upon the upper bounds for planar graphs of high girth, less is known about planar graphs of low girth. As mentioned in the introduction, Albertson et al. [1] show the star chromatic number for planar graphs 
is somewhere from 10 to 20. The gap is also wide for bipartite planar graphs. In [7], it is shown that bipartite planar graphs can be star colored using 14 colors, and an example of a bipartite planar graph requiring 8 colors to star color is given.

\section{Acknowledgements}

The author would like to thank André Kündgen for many helpful suggestions, Mike Albertson for planting a seed by bringing the configuration in Claim 1, Theorem 3.1 to our attention. Additionally, the author would like to thank the referee for many comments that substantially improved the presentation of the paper.

\section{References}

[1] M.O. Albertson, G.G. Chappell, H.A. Kierstead, A. Kündgen and R. Ramamurthi, Coloring with no 2-colored $P_{4}$ 's, Electronic J. of Combinatorics, 11 (2004), \#R26.

[2] M.O. Albertson, Personal Communication, (2007).

[3] O.V. Borodin, On acyclic colorings of planar graphs, Discrete Math., 25 (1979), no. 3, 211-236.

[4] O.V. Borodin, A.V. Kostochka, D.R. Woodall, Acyclic colourings of planar graphs with large girth. J. London Math. Soc. (2) 60 (1999), 344-352.

[5] B. Grünbaum, Acyclic colorings of planar graphs, Israel J. Math., 14 (1973), 390-408.

[6] H. Grötzsch, Ein Dreifarbensatz fü dreikreisfreie Netze auf der Kugel. Wiss Z. Martin-Luther-U., Halle-Wittenberg, Math.-Nat. Reihe 8 (1959), 109-120.

[7] H.A. Kierstead, A. Kündgen, C. Timmons, Star coloring planar bipartite graphs, J. Graph Theory, to appear.

[8] A. Kündgen, C. Timmons, Star coloring planar graphs from small lists, submitted.

[9] J. Nešetřil and P. Ossona de Mendez, Colorings and homomorphisms of minor closed classes, Discrete and Computational Geometry: The Goodman-Pollack Festschrift (ed. B. Aronov, S. Basu, J. Pach, M. Sharir), Springer-Verlag 2003, 651-664.

[10] C. Timmons, Star Coloring Planar Graphs, Master's Thesis, California State University San Marcos (2007). 